\title{
Quantum Andreev effect in two-dimensional HgTe/CdTe quantum well/superconductor systems
}

\author{
Qing-Feng Sun, ${ }^{1, *}$ Yu-Xian Li, ${ }^{2}$ Wen Long, ${ }^{3}$ and Jian Wang ${ }^{4, \dagger}$ \\ ${ }^{1}$ Institute of Physics, Chinese Academy of Sciences, Beijing 100190, China \\ ${ }^{2}$ College of Physics, Hebei Normal University, Shijiazhuang 050016, China \\ ${ }^{3}$ Department of Physics, Capital Normal University, Beijing 100048, China \\ ${ }^{4}$ Department of Physics and The Center of Theoretical and Computational Physics, The University of Hong Kong, Hong Kong, China
}

(Received 16 July 2010; revised manuscript received 23 January 2011; published 11 March 2011)

\begin{abstract}
The Andreev reflection (AR) in 2D HgTe/CdTe quantum well-superconductor hybrid systems is studied. A quantized AR with AR coefficient equal to one is predicted, which is due to the multi-Andreev reflection near the interface of the hybrid system. Importantly, this quantized AR is not only universal, i.e., independent of any system parameters and quality of the coupling of the hybrid system, it is also robust against disorder as well. As a result of this quantum Andreev effect, the conductance exhibits a quantized plateau when the external bias is less the superconductor gap.
\end{abstract}

DOI: 10.1103/PhysRevB.83.115315

PACS number(s): 74.45.+c, 73.23.-b, 85.75.-d

Recently, the topological insulator (TI), a new state of matter, has attracted a lot of theoretical and experimental attention. ${ }^{1-12}$ The TI has an insulating energy gap in the bulk states, but it has exotic gapless metallic states on its edges or surfaces. The TI is first predicted in two-dimensional (2D) systems, e.g., the graphene and $\mathrm{HgTe} / \mathrm{CdTe}$ quantum well $(\mathrm{QW}){ }^{2,3}$ The 2D TI has the gapless helical edge states and exhibits the quantum spin Hall effect. This helical edge states, with the opposite spins on a given edge or opposite edges for a given spin direction containing opposite propagation directions, are topologically protected and are robust against all time-reversal-invariant impurities. Soon after that, people also found the TI in three-dimensional (3D) materials, e.g., $\mathrm{Bi}_{1-x} \mathrm{Sb}_{x}, \mathrm{Bi}_{2} \mathrm{Se}_{3}$, etc. ${ }^{4-6}$ On the experimental side, the TI in $\mathrm{HgTe} / \mathrm{CdTe} \mathrm{QW}, \mathrm{Bi}_{1-x} \mathrm{Sb}_{x}, \mathrm{Bi}_{2} \mathrm{Se}_{3}$, and so on, have been successfully confirmed. ${ }^{4,5,7,8}$

The Andreev reflection (AR) which was found about 50 years ago is an important transport process. ${ }^{13}$ The AR occurs near the interface of a conductor and a superconductor, in which an incident electron from the metallic side is reflected as a hole and a Cooper pair is created in the superconductor. When the bias is smaller than the superconductor gap, the conductance of the conductor-superconductor hybrid device is mainly determined by the AR. ${ }^{14}$ For a perfect conductorsuperconductor interface, the probability of the AR can reach one. However, the AR coefficient is usually very small due to various scattering mechanisms such as the contact potential of the interface, the impurities, the mismatch of the density of states of the conductor and superconductor, and so on. Although the AR has been extensively investigated in various conductor-superconductor hybrid systems, up to now, people has not observed a robust quantized AR that holds for various system parameters and sustains in the presence of a variety of scattering mechanisms.

In this article, we study the AR in a 2D TI-superconductor (TI-S) hybrid system. We found that in this system the AR is quantized with AR coefficient being one, because of the multi-Andreev reflection along the TI-S interface. Importantly, this quantized AR is universal and robust. The quantization of AR persists regardless of the system parameters such as the Fermi energy, energy of the incident electron, and the size of the system. It is also robust against the presence of impurities and contact potential of the interface. Due to the quantum Andreev effect, the conductance exhibits the plateau with the plateau value being $4 e^{2} / h\left(2 e^{2} / h\right)$ for the two-terminal (fourterminal) TI-S hybrid system.

Since the TI phase in $\mathrm{HgTe} / \mathrm{CdTe} \mathrm{QW}$ has been experimentally realized, ${ }^{7,8}$ we shall focus on the $\mathrm{HgTe} / \mathrm{CdTe} \mathrm{QW}$ in the following calculation. The results will be the same for other 2D TI systems. We consider two HgTe/CdTe QW-superconductor hybrid devices as shown in Fig. 1: one is a ribbon of $\mathrm{HgTe} / \mathrm{CdTe}$ QW coupled to a semi-infinite superconducting lead which is referred as the two-terminal device and the other is a cross of $\mathrm{HgTe} / \mathrm{CdTe} \mathrm{QW}$ coupled to a superconducting lead which is referred as the four-terminal device. The advantage of the four-terminal device is that the "trajectories" of the incident electron and reflected hole can be clearly shown.

The hybrid devices are described by the Hamiltonian $H=$ $H_{\mathrm{TI}}+H_{S}+H_{C}$, where $H_{\mathrm{TI}}, H_{S}$, and $H_{C}$ are the Hamiltonian of the $\mathrm{HgTe} / \mathrm{CdTe} \mathrm{QW}$, superconducting lead, and the coupling between them, respectively. By discretizing spatial coordinates of the continuous effective Hamiltonian $H_{\mathrm{TI}}$ in Ref. 3 and using the Nambu representation, ${ }^{15}$ the Hamiltonian $H_{\mathrm{TI}}$ is given by ${ }^{16}$

$$
H_{\mathrm{TI}}=\sum_{\mathbf{i}} \Psi_{\mathbf{i}}^{\dagger} \breve{H}_{\mathrm{ii}} \Psi_{\mathbf{i}}+\sum_{\vec{\alpha}=(\vec{\delta} x, \vec{\delta} y), \mathbf{i}} \Psi_{\mathbf{i}}^{\dagger} \breve{H}_{\mathrm{i} \vec{\alpha}} \Psi_{\mathbf{i}+\vec{\alpha}}+\text { H.c., }
$$

where $\mathbf{i}=\left(i_{x}, i_{y}\right)$ is the site index, $\vec{\delta} x$ and $\vec{\delta} y$ are unit vectors along $x$ and $y$ directions, $\Psi_{\mathbf{i}}=\left(a_{\mathbf{i}}, b_{\mathbf{i}}, c_{\mathbf{i}}^{\dagger}, d_{\mathbf{i}}^{\dagger}\right)^{T}$, and $a_{\mathbf{i}}, b_{\mathbf{i}}, c_{\mathbf{i}}, d_{\mathbf{i}}$ are annihilation operators of electron on the site $\mathbf{i}$ at the states $|s, \uparrow\rangle,\left|p_{x}+i p_{y}, \uparrow\right\rangle,|s, \downarrow\rangle$, and $-\mid p_{x}-i p_{y}$, $\downarrow$. In Eq. (1), $\breve{H}_{\mathrm{ii} / \mathrm{i} / \vec{\delta} x / / \vec{\delta} y}=\left(\begin{array}{cc}H_{\mathrm{ii} / / \vec{\delta} x} / \mathrm{i} \vec{\delta} y & \mathbf{0} \\ \mathbf{0} & -H_{\mathrm{ii} / \mathrm{i} \delta} x / \mathrm{i} \vec{\delta} y\end{array}\right)$ are the $4 \times 4$ matrix Hamiltonian, where $H_{\mathrm{ii}}=\left(\begin{array}{cc}E_{s} & 0 \\ 0 & E_{p}\end{array}\right), H_{\mathrm{i} \vec{\delta} x}=$ $\left(\begin{array}{cc}t_{s s} & -i t_{s p} \\ -i t_{s p} & t_{p p}\end{array}\right)$, and $H_{\mathrm{i} \vec{\delta} y}=\left(\begin{array}{cc}t_{s s} & -t_{s p} \\ t_{s p} & t_{p p}\end{array}\right)$, with $E_{s / p}=C \pm M-$ $E_{F}-4(D \pm B) / a^{2}, t_{s s / p p}=(D \pm B) / a^{2}, t_{s p}=A / 2 a$. Here $E_{F}$ is the Fermi energy (pinned by superconductor condensate), $a$ is the lattice constant, and $A, B, C, D$, and $M$ are the system's parameters which can be experimentally controlled. The Hamiltonian $H_{S}$ of the superconducting lead is $H_{S}=\Sigma_{\mathbf{k}, \sigma} \epsilon_{\mathbf{k}} a_{S \mathbf{k} \sigma}^{\dagger} a_{S \mathbf{k} \sigma}+\Sigma_{\mathbf{k}} \Delta\left(a_{S \mathbf{k} \uparrow}^{\dagger} a_{S-\mathbf{k} \downarrow}^{\dagger}+\right.$ H.c. $)$ where $\Delta$ 

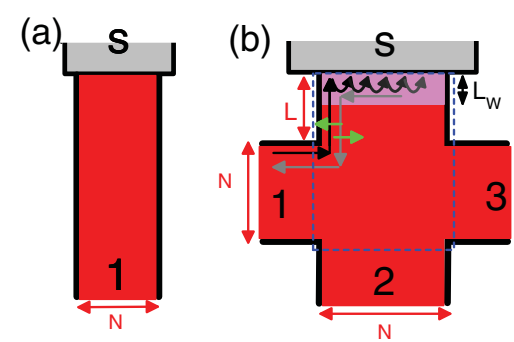

FIG. 1. (Color online) (a) and (b) are the schematic diagram for the two-terminal and four-terminal devices.

is the superconductor gap and $a_{S \mathbf{k} \sigma}^{\dagger}\left(a_{S \mathbf{k} \sigma}\right)$ is the creation (annihilation) operators in the superconducting lead with the momentum $\mathbf{k}=\left(k_{x}, k_{y}\right)$. Here we consider the general $s$-wave superconductor. The coupling Hamiltonian $H_{C}$ is $H_{C}=\sum_{\mathbf{i}}\left(a_{S i_{x} \uparrow}^{\dagger}, a_{S i_{x} \downarrow}\right) \mathbf{t}_{S} \Psi_{\mathbf{i}}+$ H.c., where the operator $a_{S i_{x} \sigma}=$

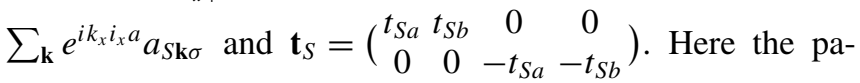
rameters $t_{S a}$ and $t_{S b}$ are the coupling strengths between the superconductor and $\mathrm{HgTe} / \mathrm{CdTe} \mathrm{QW}$, which depends on the interface's contact potential and the quality of the coupling in the experiment.

By using the Green's functions, the charge current $I_{n e}$ and spin current $I_{s e}$ from the $n$th terminal of the $\mathrm{HgTe} / \mathrm{CdTe}$ QW flowing into the device are $I_{n e}=e\left(I_{n \uparrow}+I_{n \downarrow}\right)$ and $I_{n s}=$ $(\hbar / 2)\left(I_{n \uparrow}-I_{n \downarrow}\right)$, where ${ }^{17,18}$

$$
\begin{aligned}
I_{n \sigma}= & \frac{1}{h} \int d E\left\{\sum_{m} T_{n m \sigma}\left(f_{n \sigma}-f_{m \sigma}\right)+T_{n s \sigma}\left(f_{n \sigma}-f_{s}\right)\right. \\
& \left.+\sum_{m} T_{n \sigma, m \bar{\sigma}}^{A}\left(f_{n \sigma}-f_{m \bar{\sigma}}\right)\right\} .
\end{aligned}
$$

Here $\bar{\sigma}=\downarrow, \uparrow$ for $\sigma=\uparrow, \downarrow, f_{n \uparrow / \downarrow}(E)=f\left(E \mp e V_{n}\right)$, and $f_{s}(E)=f(E)$, with $f(E)$ being the Fermi distribution function and $V_{n}$ being the voltage of the terminal $n$. In Eq. (2), $T_{n m \sigma}(E)=\operatorname{Tr}\left\{\Gamma_{n \sigma} \mathbf{G}_{\sigma \sigma}^{r} \Gamma_{m \sigma} \mathbf{G}_{\sigma \sigma}^{a}\right\}$ and $T_{n s \sigma}(E)=$ $\operatorname{Tr}\left\{\Gamma_{n \sigma}\left[\mathbf{G}^{r} \Gamma_{s} \mathbf{G}^{a}\right]_{\sigma \sigma}\right\}$ are, respectively, the normal transmission coefficient from the terminal $n$ to the terminal $m$ and to the superconductor terminal, and $T_{n \sigma, m \bar{\sigma}}^{A}(E)=\operatorname{Tr}\left\{\Gamma_{n \sigma} \mathbf{G}_{\sigma \bar{\sigma}}^{r} \Gamma_{m \bar{\sigma}} \mathbf{G}_{\bar{\sigma} \sigma}^{a}\right\}$ is the AR coefficient with the incident electron from the terminal $n$ and the reflected hole going to the terminal $m$. The linewidth function $\Gamma_{n / s}(E)=i\left[\Sigma_{n / s}^{r}-\left(\Sigma_{n / s}^{r}\right)^{\dagger}\right]$ and the Green's functions $\mathbf{G}^{r / a}(E)$ can be calculated from $\mathbf{G}^{r}(E)=$ $\left[\mathbf{G}^{a}(E)\right]^{\dagger}=\left\{E-\mathbf{H}_{c e n}-\sum_{n} \Sigma_{n}^{r}-\Sigma_{s}^{r}\right\}^{-1}$, where $\mathbf{H}_{c e n}$ is the Hamiltonian of the scattering region as shown in Fig. 1 (dotted region). The self-energy functions $\Sigma_{n}^{r}(E)$ and $\Sigma_{s}^{r}(E)$ due to terminals of the TI and superconducting lead can be calculated as in Refs. [ 17-20]. In the following numerical calculations, we choose the parameters from the realistic materials ${ }^{7}$ : (1) The HgTe/CdTe QW's parameters are $A=364.5 \mathrm{meV} \mathrm{nm}$, $B=-686 \mathrm{meV} \mathrm{nm}^{2}, C=0$, and $D=-512 \mathrm{meV} \mathrm{nm}^{2}$. (2) The superconductor's parameters are the gap energy $\Delta=$ $1 \mathrm{meV}$. The lattice constant $a$ is set to $5 \mathrm{~nm}$ and the TI-S coupling strengths are taken $t_{S a}=t_{S b} \equiv t$.

We first study the two-terminal system. Due to the timereversal symmetry and the $C_{2}$ symmetry around the $y$ axis, the AR coefficients have the properties $T_{1 \uparrow, 1 \downarrow}^{A}(E)=T_{1 \downarrow, 1 \uparrow}^{A}(E) \equiv$

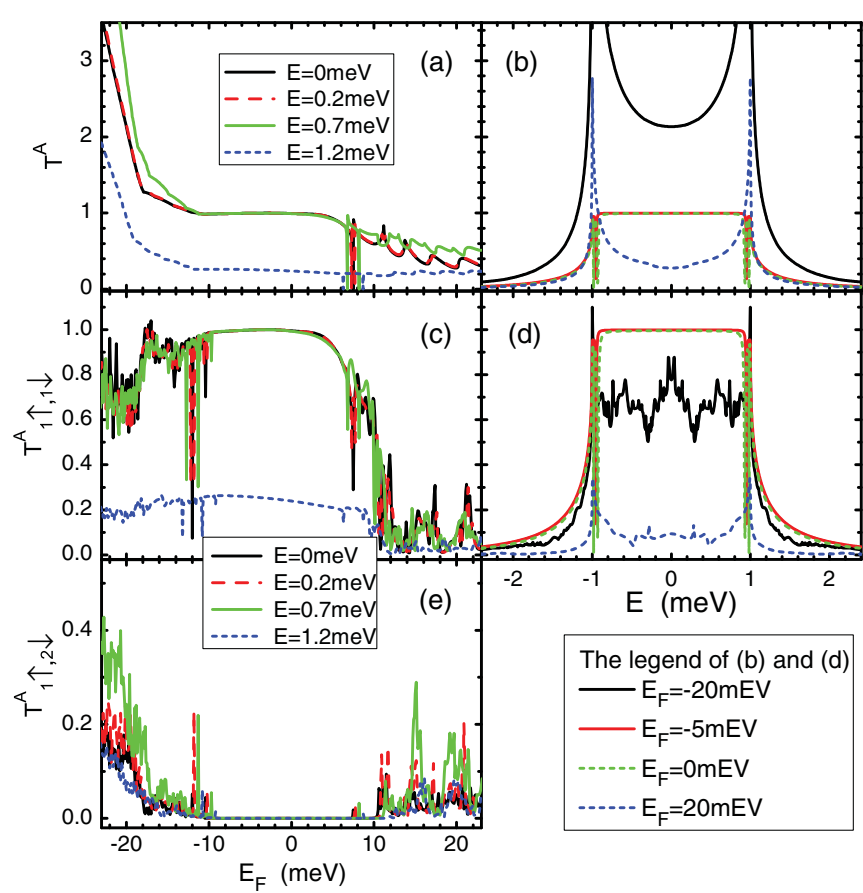

FIG. 2. (Color online) (a) and (b) The AR coefficient $T^{A}$ vs. $E_{F}$ (a) and energy $E$ (b) in the two-terminal device. (c), (d), and (e) $T_{1 \uparrow, 1 \downarrow}^{A}$ vs. $E_{F}$ (c), $T_{1 \uparrow, 1 \downarrow}^{A}$ vs. the energy $E(\mathrm{~d})$, and $T_{1 \uparrow, 2 \downarrow}^{A}$ vs. $E_{F}$ (e) in the four-terminal device with the length $L=750 \mathrm{~nm}$. The other parameters in (a)-(e) are the width $N=300 \mathrm{~nm}$, coupling strength $t=3 \mathrm{meV}$, and $M=-10 \mathrm{meV}$.

$T^{A}(E)$ and $T^{A}(E)=T^{A}(-E)$. Figures 2(a) and 2(b) show the AR coefficient $T^{A}$ versus the Fermi energy $E_{F}$ and energy $E$ of the incident electron. Now the parameter $M$ is set $-10 \mathrm{meV}$ so that the $\mathrm{HgTe} / \mathrm{CdTe}$ QW is in the TI phase. ${ }^{3}$ Figures 2(a) and 1(b) clearly show that $T^{A}$ exhibits a plateau with the plateau value equal to one as long as $E_{F}$ is in the bulk gap of TI and the energy $E$ within the superconductor gap $\Delta$. Since there is only one transmission channel (i.e., the edge state) when $E_{F}$ is in the bulk gap, $T^{A}=1$ means that the incident electron is completely Andreev reflected. It is remarkable that the quantum Andreev effect occurs in such a wide range of $E_{F}$ and $E$, while in all previous metal-superconductor hybrid systems the resonant condition $T^{A}=1$ occurs only at certain set of parameters. Figure 2(a) also shows that when $E_{F}$ is out of the bulk gap, there is no plateau in the AR coefficient $T^{A}$ and $T^{A}$ depends on both $E_{F}$ and $E$. For $E_{F}<M, T^{A}$ can be larger than one (see Fig. 2(a) because there are many transmission channels. However, the AR coefficient for each transmission channel is still much smaller than one.

In order to reveal the nature of quantum Andreev effect, we will study the four-terminal device. In the four-terminal device, the AR coefficient $T_{n \uparrow, m \downarrow}^{A}$ has $3 \times 3=9$ elements, so the trajectories of the incident electron and reflected hole can clearly be shown. When the HgTe/CdTe QW is in the TI regime, only $T_{1 \uparrow, 1 \downarrow}^{A}$ is nonzero and the other eight elements are zero. In particular, as soon as the energy $|E|<\Delta$ and $M<$ $E_{F}<-M$, the quantized plateau with $T_{1 \uparrow 1 \downarrow}^{A}=1$ emerges and this plateau is independent of $E_{F}$ and $E$ (see Figs. 2(c) and 2(d)). These results can be understood with the help of 
helical edge states, in which the spin-up and spin-down carriers move along the edge of the $\mathrm{HgTe} / \mathrm{CdTe} \mathrm{QW}$ in clockwise and counterclockwise directions, respectively. ${ }^{1,3}$ We consider the case of the spin-up electron coming from terminal-1 when the energy $|E|<\Delta$. As shown in Fig. 1(b), two reflection processes occur at the TI-S interface: (1) It can be Andreev reflected back as a spin-down hole to the same terminal which will contribute to $T_{1 \uparrow, 1 \downarrow}^{A}$. (2) It can also be normal reflected as an electron (spin up) along the TI-S interface, eventually to terminal-3. Note that the normal reflection as an electron back to terminal-1 is prohibited by the time-reversal invariance and the helical edge states being a pair of Kramer states. The reflected electron traveling to terminal-3 has to go along the TI-S interface since the only available transmission channel is the edge state. This results in a reflection again at the TI-S interface where part of the electron is Andreev reflected as the hole back to terminal- 1 and the rest is normal reflected as electron toward terminal-3. As this continues, multireflections occur as normal electron traverses along the TI-S interface and eventually the transmission probability $T_{13}$ becomes zero if the TI-S interface is long enough. Clearly, it is this multi-AR that gives rise to the quantum Andreev effect with the quantized AR $T_{1 \uparrow, 1 \downarrow}^{A}=1$. Furthermore, we have three observations: (i) If the energy $|E|>\Delta$, all AR coefficients decrease as usual (see Fig. 2(d)), ${ }^{21}$ because of the occurrence of the normal tunneling from TI to the superconductor. (ii) When $E_{F}$ is out of the bulk gap, all AR coefficients have the same behavior: AR coefficient for each transmission channel is in general small and strongly depends on $E_{F}$ and $E$ (see Figs. 2(c), 2(d), and 2(e)). (iii) For the spin-down incident carrier, it is easy to show that $T_{3 \downarrow, 3 \uparrow}^{A}$ has the quantized plateau similar to $T_{1 \uparrow, 1 \downarrow}^{A}$.

Next, we study how the quantized AR is affected by the system parameters. Figure 3 shows the AR coefficient $T_{1 \uparrow, 1 \downarrow}^{A}$ versus the width $N$ of the $\mathrm{HgTe} / \mathrm{CdTe} \mathrm{QW}$ ribbon and the coupling strength $t$. The results show that the quantization of AR persists for a broad range of the coupling strength $t$. In addition, the wider the width $N$, the broader the quantization plateau is. For $N=1000 \mathrm{~nm}$, the AR quantization plateau can sustain when $t$ varies nearly one order of magnitude. Finally, with the increase of the width $N$, the AR coefficient $T_{1 \uparrow, 1 \downarrow}^{A}$ rises monotonously before it reaches quantized value due to the fact that the incident electron has more chance of multi-AR for the longer TI-S interface. These results show the universal feature of the quantum Andreev effect: it is independent of the system parameters.

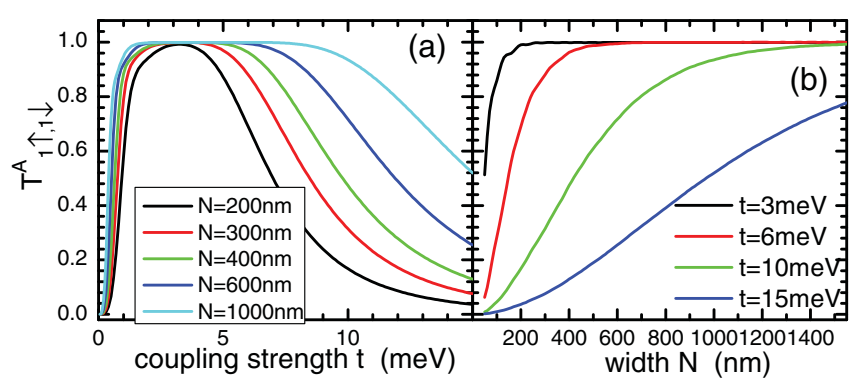

FIG. 3. (Color online) $T_{1 \uparrow, 1 \downarrow}^{A}$ vs. $t$ (a) and width $N$ (b) in the four-terminal device with $E_{F}=-5 \mathrm{meV}, E=0.1 \mathrm{meV}, M=-$ $10 \mathrm{meV}$, and length $L=750 \mathrm{~nm}$.
Is the quantized AR robust against the disorder? To answer this question, we consider the on-site Anderson disorder in a region near the TI-S interface [see the light gray (red) region in Fig. 1(b)]. Because of the disorder, an extra on-site term $\Psi_{\mathbf{i}}^{\dagger} \breve{w}_{\mathbf{i}} \Psi_{\mathbf{i}}$ is added on each site $\mathbf{i}$ in the disorder region, where $\breve{w}_{\mathbf{i}}$ is the $4 \times 4$ diagonal matrix with diagonal elements $\left(w_{\mathbf{i}}, w_{\mathbf{i}},-w_{\mathbf{i}},-w_{\mathbf{i}}\right) . w_{\mathbf{i}}$ is assumed uniformly distributed in the range $[-W / 2, W / 2]$ with the disorder strength $W$. Figure 4 shows the AR coefficients $T_{1 \uparrow, 1 \downarrow / 2 \downarrow}^{A}$ and its fluctuation versus $E_{F}$ and $E$. The results show that the quantized AR plateau in $T_{1 \uparrow, 1 \downarrow}^{A}$ is very robust: the AR plateau can persist and its fluctuation $\operatorname{rms}\left(T_{1 \uparrow, 1 \downarrow}^{A}\right)$ is zero for the disorder strength $W$ up to $100 \mathrm{meV}$ because of the helical edge states being very robust. Upon further increasing of $W$ from $100 \mathrm{meV}, T_{1 \uparrow, 1 \downarrow}^{A}$ starts to decrease and the fluctuation becomes nonzero because at large disorders the system reaches the diffusive regime and the helical edge states are destroyed. Hence, as long as the edge state is survived, disorder has no effect on the quantum Andreev effect.

Let us investigate the conductance $G_{n e}\left(G_{n e} \equiv d I_{n e} / d V\right)$ and spin conductance $G_{n s}\left(G_{n s} \equiv d I_{n s} / d V\right)$. We set the biases of the $\mathrm{HgTe} / \mathrm{CdTe} \mathrm{QW}$ terminals, $V_{1}=V_{2}=V_{3} \equiv V$, and the superconductor-terminal bias $V_{s}=0$. Figure 5(a) shows the conductance $G_{1 e}$ and spin conductance $G_{1 s}$ versus the bias $V$ for the four-terminal system. When $E_{F}$ is inside the bulk gap, $G_{1 e}$ (in the unit $e^{2} / h$ ) is exactly equal to $G_{1 s}$ (in the unit $e / 4 \pi$ ) since only the spin-up electron traverses from the terminal-1 to the TI-S interface with the spin-down hole Andreev reflected back. In particular, when $V<\Delta / e$, a (spin) conductance plateau emerges with the plateau value $2 e^{2} / h$ $(e / 2 \pi)$ because of the quantized $\mathrm{AR}$ with $T_{1 \uparrow, 1 \downarrow}^{A}=1$. Here we emphasize that like the quantized AR this conductance plateau is also universal (i.e., it is independent of the system parameters and the quality of TI-S coupling) and robust against the disorder. Since the TI phase in $\mathrm{HgTe} / \mathrm{CdTe} \mathrm{QW}$ has been realized experimentally, ${ }^{7,8}$ this predicted quantum Andreev effect with quantized conductance plateau should

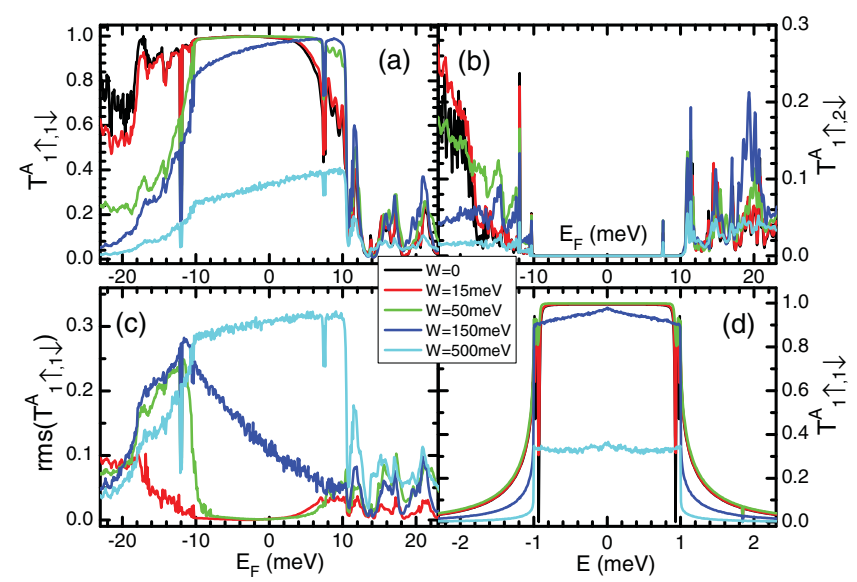

FIG. 4. (Color online) (a), (b), and (c) are $T_{1 \uparrow, 1 \downarrow}^{A}$ (a), $T_{1 \uparrow, 2 \downarrow}^{A}$ (b), and $\operatorname{rms}\left(T_{1 \uparrow, 1 \downarrow}^{A}\right)$ (c) vs. $E_{F}$ with the energy $E=0.1 \mathrm{meV}$ for different disorder strengths $W$. (d) is $T_{1 \uparrow, 1 \downarrow}^{A}$ vs. $E$ with $E_{F}=0$. The length of the disorder region $L_{W}=150 \mathrm{~nm}$ and the other parameters are the same as Fig. 2. Here all curves are averaged over up to 2000 random configurations. 


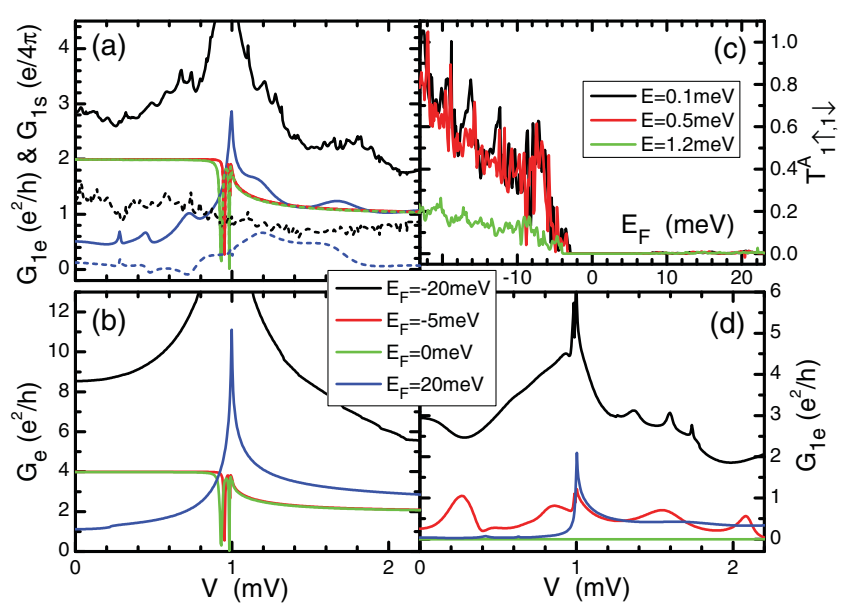

FIG. 5. (Color online) (a) is the conductance $G_{1 e}$ (solid curves) and spin conductance $G_{1 s}$ (dotted curves) vs. the bias $V$ for different $E_{F}$ [with the legend being the same in (b)] for the four-terminal device. (b) is the conductance $G_{e}$ vs. the bias $V$ for the two-terminal device. (c) and (d) are $T_{1 \uparrow, 1 \downarrow}^{A}$ vs. $E_{F}$ (c) and conductance $G_{1 e}$ vs. bias $V$ (d) for the four-terminal device with the positive $M=2 \mathrm{meV}$. The other parameters in (a)-(d) are the same as Fig. 2.

not be difficult to observe using the present technology. On the other hand, when $E_{F}$ is out of the bulk gap, both $G_{1 e}$ and $G_{1 s}$ are not equal and they are sensitive to the system parameters. Finally, we comment on following two points: (i) If $\mathrm{HgTe} / \mathrm{CdTe} \mathrm{QW}$ is in the normal state (i.e., $M>0$ ), no plateau emerges for both the AR coefficient $T_{n \uparrow, m \downarrow}^{A}$ and conductance $G_{n e}$ (see Figs. 5(c) and 5(d)), similar to the ordinary conductor-superconductor hybrid system. (ii) For the two-terminal device with the $\mathrm{HgTe} / \mathrm{CdTe} \mathrm{QW}$ in TI regime, the conductance $G_{e}$ also exhibits the quantum Andreev effect with quantized AR conductance $4 e^{2} / h$ at $V<\Delta / e$ (see Fig. 5(b)). However, the spin conductance $G_{s}$ is exactly zero due to the fact that the left and right edges of the $\mathrm{HgTe} / \mathrm{CdTe} \mathrm{QW}$ ribbon carry the same current but opposite spin current.

In summary, we predict a quantum Andreev effect in the 2D TI-S hybrid system, in which the AR coefficient is quantized with the value one. Importantly, the quantized AR plateau is independent of various system parameters and the quality of TI-S coupling, and it is also robust against the disorders. Due to the excellent properties of the quantum Andreev effect and the 2D TI having experimentally been realized in $\mathrm{HgTe} / \mathrm{CdTe}$ quantum well, so it should be easy to observe it using the present technology. In addition, due to the quantized AR, the conductance and spin conductance versus the bias also exhibit the quantized plateau when the bias is within the superconductor gap.

Recently, we noticed that the prefect AR with the AR coefficient $T^{A}=1$ is also addressed by Adroguer et al. using the one-dimensional model. ${ }^{22}$ Here we consider the two-dimensional TI-S device, the full band structures of the $\mathrm{TI}$ and superconductor are involved, and in particular we point out that the AR plateau is universal and robust in contrast to the conclusion of Ref. 22.

\section{ACKNOWLEDGMENTS}

This work was financially supported by NSFC under Grant Nos. 10974236, 10974043, and 11074174, and a RGC Grant (No. HKU 704308P) from the government of HKSAR. *sunqf@aphy.iphy.ac.cn

†jianwang@hkusua.hku.hk

${ }^{1}$ J. M. Moore, Nature (London) 464, 104 (2010).

${ }^{2}$ C. L. Kane and E. J. Mele, Phys. Rev. Lett. 95, 146802 (2005); 95, 226801 (2005).

${ }^{3}$ B. A. Bernevig, T. L. Hughes, and S. C. Zhang, Science 314, 1757 (2006).

${ }^{4}$ D. Hsieh, D. Qian, L. Wray, Y. Xia, Y. S. Hor, R. J. Cava, and M. Z. Hasan, Nature (London) 452, 970 (2008); D. Hsieh, Y. Xia, D. Qian, L. Wray, J. H. Dil, F. Meier, J. Osterwalder, L. Pattey, J. G. Checkelsky, N. P. Ong, A. V. Fedorov, H. Lin, A. Bansil, D. Grauer, Y. S. Hor, R. J. Cava, and M. Z. Hasan, ibid. 460, 1101 (2009).

${ }^{5}$ Y. L. Chen, J. G. Analytis, J.-H. Chu, Z. K. Liu, S.-K. Mo, X. L. Qi, H. J. Zhang, D. H. Lu, X. Dai, Z. Fang, S. C. Zhang, I. R. Fisher, Z. Hussain, and Z.-X. Shen, Science 325, 178 (2009); T. Zhang, P. Cheng, X. Chen, J.-F. Jia, X. Ma, K. He, L. Wang, H. Zhang, X. Dai, Z. Fang, X. Xie, and Q.-K. Xue, Phys. Rev. Lett. 103, 266803 (2009).

${ }^{6}$ L. Fu, C. L. Kane, and E. J. Mele, Phys. Rev. Lett. 98, 106803 (2007); H. Zhang, C.-X. Liu, X.-L. Qi, X. Dai, Z. Fang, and S.-C. Zhang, Nat. Phys. 5, 438 (2009).

${ }^{7}$ M. König, S. Wiedmann, C. Brüne, A. Roth, H. Buhmann, L. W. Molenkamp, X.-L. Qi, and S.-C. Zhang, Science 318, 766 (2007); M. König, H. Buhmann, L. W. Molenkamp, T. L. Hughes, C. X. Liu, X. L. Qi, and S. C. Zhang, J. Phys. Soc. Jpn. 77, 031007 (2008).
${ }^{8}$ A. Roth, C. Brüne, H. Buhmann, L. W. Molenkamp, J. Maciejko, X.-L. Qi, and S.-C. Zhang, Science 325, 294 (2009).

${ }^{9}$ H. Peng, K. Lai, D. Kong, S. Meister, Y. Chen, X.-L. Qi, S.-C. Zhang, Z.-X. Shen, and Y. Cui, Nature Materials 9, 225 (2010).

${ }^{10}$ H. Jiang, S. G. Cheng, Q.-F. Sun, and X. C. Xie, Phys. Rev. Lett. 103, 036803 (2009).

${ }^{11}$ Q.-F. Sun and X. C. Xie, Phys. Rev. Lett. 104, 066805 (2010).

${ }^{12}$ M. Guigou and J. Cayssol, Phys. Rev. B 82, 115312 (2010).

${ }^{13}$ A. F. Andreev, Sov. Phys. JETP 19, 1228 (1964).

${ }^{14}$ K. Kang, Phys. Rev. B 57, 11891 (1998); Physica E 5, 36 (1999).

${ }^{15}$ In fact, from the continuous effective Hamiltonian $H_{\mathrm{TI}}$ and using the Nambu representation, this problem can also be solved. For example, one can obtain the transmission and AR coefficients by solving the scattering wave function in the real space with the matching boundary conditions, as done in Ref. 22 and Q.-F. Sun and X. C. Xie, Phys. Rev. B 71, 155321 (2005). No matter which methods are applied, the results are the same, because the present system can exactly be solved.

${ }^{16}$ H. Jiang, L. Wang, Q.-F. Sun, and X. C. Xie, Phys. Rev. B 80, 165316 (2009)

${ }^{17}$ Q.-F. Sun and X. C. Xie, J. Phys.: Condens. Matter 21, 344204 (2009).

${ }^{18}$ S.-G. Cheng, Y. X. Xing, J. Wang, and Q.-F. Sun, Phys. Rev. Lett. 103, 167003 (2009). 
${ }^{19}$ M. P. Lopez Sancho, J. M. Lopez Sancho, and J. Rubio, J. Phys. F: Met. Phys. 14, 1205 (1984); ibid. 15, 851 (1985).

${ }^{20}$ Q.-F. Sun, J. Wang, and T.-H. Lin, Phys. Rev. B 59, 3831 (1999); 59, 13126 (1999).
${ }^{21}$ G. E. Blonder, M. Tinkham, and T. M. Klapwijk, Phys. Rev. B 25, 4515 (1982).

${ }^{22}$ P. Adroguer, C. Grenier, D. Carpentier, J. Cayssol, P. Degiovanni, and E. Orignac, Phys. Rev. B 82, 081303(R) (2010). 